\title{
Total Dose Effect on Ferroelectric PZT Capacitors used as Non-Volatile Storage Elements*
}

\author{
R. A. Moore, J. Benedetto, and B. J. Rod \\ Army Research Laboratory \\ Adelphi, MD 20783
}

\begin{abstract}
This paper examines the effects of ionizing radiation on the retained polarization of lead zirconate titanate (PZT) thin films. The retained polarization is the key parameter in measuring the radiation tolerance of the PZT storage element in a non-volatile memory. Data from the retained polarization measurement show a larger radiation-induced degradation than has generally been reported using the traditional hysteresis loop method for measuring remanent polarization. It appears that the difference is due in part to a cycling-induced annealing effect during the hysteresis loop measurement.
\end{abstract}

\section{INTRODUCTION}

Lead zirconate titanate (PZT) is currently the only ferroelectric (FE) material used as the storage element for commercially available nonvolatile random access memories (NVRAMs) [1]. The total-dose ionizing radiation response of thin-film $P Z T$ has been studied by many groups [2-6] and it has generally been found to be radiation hard to extremely large doses (up to 100 $\operatorname{Mrad}(\mathrm{Si})$ ). The figure of merit used to evaluate the radiation response in these studies was typically the remanent polarization as measured by a hysteresis loop in a Sawyer-Tower type circuit [7], although one group reported results using a series of voltage pulses to the switchable polarization [3]

When a FE film is used as a memory element in a NVRAM, the retained polarization, not the remanent polarization, becomes the key parameter. The retained polarization measurement is essentially identical to a single write/read cycle of a given NV memory bit and can be used to determine the size of the memory window (the difference between a stored "1" and a stored " 0 ") that would be available to a memory sense amp. As is discussed in more detail in the next section, the retained polarization measurement and the hysteresis loop differ because of significant "non- retained" polarization contribution in the continuous hysteresis loop measurement.

Since the retained polarization is more representative of how PZT responds in a NVRAM, we examine the effect of ionizing radiation on the retained polarization. This allows us to evaluate the memory window degradation as a function of total dose and to evaluate the expected performance of a PZT-based NVRAM in an ionizing radiation environment.

\section{MATERIALS}

Ferroelectric materials have the unique property of maintaining an electrical polarization without applied bias. The orientation of the polarization can be set by the application of an external electric field and can be electrically sensed, destructively, by applying a field across the capacitor and noting the size of the resulting current pulse through it. If the orientation was already in the same direction as the applied electric field, there will be a relatively small resulting current pulse; if the orientation was opposite, there will be a relatively large current pulse. One can arbitrarily assign one polarization orientation as denoting a memory state "1" and the opposite orientation as denoting a " 0 " thus enabling a capacitor of an FE material to become a simple memory storage cell.

The polarization orientation is generally sensed by connecting one lead of the FE capacitor to a larger linear (non-ferroelectric) capacitor, which acts as a current integrator such that the current pulse from the ferroelectric capacitor appears as a voltage on the sense capacitor. This arrangement connected to a signal generator is known as a Sawyer-Tower circuit [7].

In this work, we examine the response of the electrical properties of ferroelectric PZT that are of interest for non-volatile memory applications. The test structures we used are simple capacitors with platinum (Pt) top and bottom electrodes. The capacitor dielectric layer is $2400 \AA$ of sol-gel deposited PZT (40/60). The FE sol-gel was prepared by Symetrix Corporation and the films

\footnotetext{
* This work partially supported by the Defense Nuclear Agency.
} 
were deposited (on 4 in. Si wafers) and annealed at the Army Research Laboratory at $650^{\circ} \mathrm{C}$ for $30 \mathrm{~min}$. Each capacitor had a continuous bottom electrode and a top electrode area of $1.84 \cdot 10^{-4} \mathrm{~cm}^{2}$, defined by shadow masking. The PZT layer was continuous, except at one corner of each die where the film was etched to reveal the bottom $(\mathrm{Pt})$ electrode. The parts were packaged before testing in 16 pin ceramic DIP packages without lids. We irradiated two packages separately, each with multiple FE capacitors, using $10-\mathrm{keV}$ x-rays generated by an ARACOR $4100 x$-ray tester at a dose rate of 17 $\operatorname{krad}\left(\mathrm{SiO}_{2}\right) / \mathrm{min}$.

\section{TEST Methods}

There are two different types of electrical tests typically used to measure the polarization of an FE capacitor. The most commonly used is the hysteresis loop test, in which a continuous sinusoidal voltage is applied across the FE capacitor in a Sawyer-Tower circuit (see Figure 1). When the resulting voltage across the sense capacitor is viewed as a function of the applied sinusoidal voltage, an electrical hysteresis loop results that is analogous to the magnetic hysteresis loops generated by ferromagnetic materials. The magnitude of the switched polarization is read at zero applied field and is usually denoted $2 \cdot \mathrm{Pr}$ and is called either remanent polarization or hysteresis loop polarization. This is the reported value for all of the hysteresis loop tests in this work. A sinusoidal voltage of $\pm 4 \mathrm{~V}\left(8 \mathrm{~V}_{\mathrm{pp}}\right)$ varying at a rate of $10 \mathrm{kHz}$ was applied and a sense capacitor of $47 \mathrm{nF}$ was used.

Sawyer-Tower Circuit

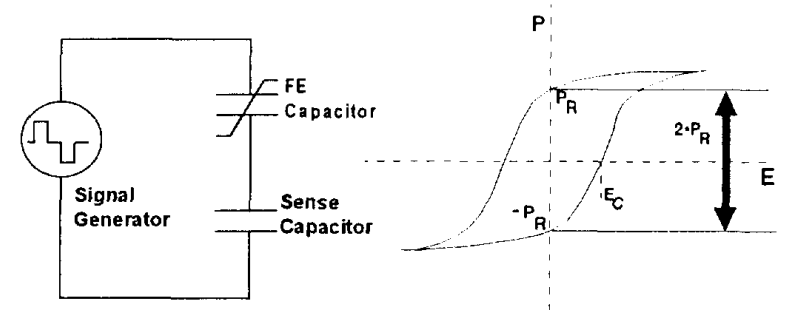

Figure 1: Schematic of Sawyer-Tower test circuit and a hysteresis loop generated when a sinusoidal waveform is produced by the signal generator. The Sawyer-Tower circuit is used for both hysteresis loop and pulse type tests.

Benedetto et al found that a component of the polarization measured by this test decays away quickly when the applied field is removed (zero external field) [8]. This component, which we call the volatile component of the polarization, typically decays away in times on the order of a millisecond. Because of this, the hysteresis loop results tend to overstate the amount of polarization available to distinguish between states in a memory application (which must retain data for more than 1 millisecond).

Therefore, we developed a second type of test, the memory pulse test, to more accurately reflect the memory storage capability of a ferroelectric capacitor. This test uses discrete, short voltage pulses to both polarize the material and to sense the orientation of the polarization. This approach therefore simulates the conditions a storage capacitor would see in a highspeed memory application. Because discrete pulses are used, the time between the initial polarization orientation (a bit is written) and when it is sensed (a bit is read) can be varied over a range from nanoseconds to years, thus allowing the volatile and non-volatile component of the polarization to be analyzed as a function of write/read delay time.

This type of test has several parameters that can be varied to monitor their effects on the FE polarization, including the time between the write pulse and the read pulse, the amplitude of the applied voltage pulses, and the width of the pulses (both read and write). Since the goal is to evaluate the films as they would perform in a high-speed memory, we set the duration of the pulses as low as possible, within the constraints of the RC time constant of the test circuit (including the relatively large area FE capacitor). In this work, all of the write/read pulse lengths are $1 \mu \mathrm{s}$ and the rise times are $100 \mathrm{~ns}$. (This is the fastest rise time we were able to use without inducing significant ringing in the test circuit.) Capacitor areas in a 1 -Mbit FE memory are projected to be $10^{-7}$ to $10^{-8} \mathrm{~cm}^{2}$ compared to $1.84 \cdot 10^{-4} \mathrm{~cm}^{2}$ for these devices. The amplitude of the applied voltages is set at $4 \mathrm{~V}$ for most tests to approximate the cell voltage available in a memory device with a $5-V$ supply voltage, although in one test of this type, the applied voltage is varied from 1 to $8 \mathrm{~V}$ to evaluate the voltage characteristic. The time between writing and reading is set at $1 \mathrm{~s}$ for all measurements, to allow sufficient time for the volatile component of the polarization to decay, except during the retention characteristic measurement where the write/read delay times are varied from $1 \mu$ s to $100 \mathrm{~s}$, to measure the volatile component of the polarization and the subsequent retention behavior.

This set of pulse tests described above ultimately seeks to find the "memory window" for an FE capacitor under the given set of conditions. The memory window is defined as the difference between the voltages recorded after reading a stored " 1 " and a stored "0". The general waveform used for this test is shown schematically in Figure 2. First, we apply a negative voltage pulse across the circuit to write the " 1 " state (the polarization orientations for the memory states were arbitrarily selected in our test) and then, after a time delay (usually $1 \mathrm{~s}$ ), a positive voltage pulse is applied to read the ' 1 ' state (the selection of a positive voltage for the read 


\section{Write '1'}

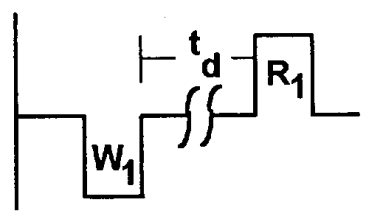

\section{Write '0'}

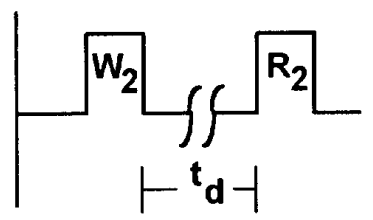

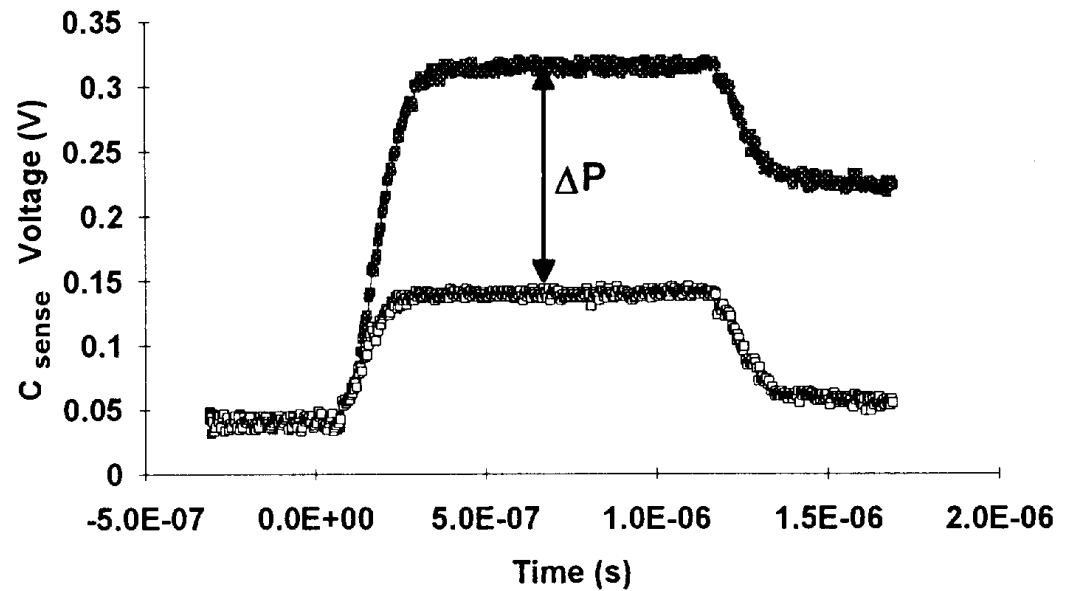

Figure 2: Schematic pulse train used in memory pulse tests and an overlay of the voltage waveforms read across the sense capacitor during a read " 1 " and a read " 0 " operation. The upper curve is from reading a ' 1 ' and the lower curve is from reading a ' 0 '.

pulse is arbitrary, but it must be consistent for all reads). Next, we apply another positive voltage pulse across the circuit to write the " 0 " state and again, after the same time delay as before, another positive voltage pulse is applied to read the "0" state. Again, the voltage waveform on the sense capacitor is recorded. Figure 2 shows the voltage waveform occurring on the sense capacitor during the two read pulses. The upper curve, measured during the first read pulse, is larger than the other because the orientation of the polarization existing before the read pulse was opposite the orientation that was imposed by the read pulse. The difference between the voltages for the two cases (write negative/read positive and write positive/read positive) represents the difference in the charge stored in the FE and is reported as a polarization. We call this quantity the retained polarization, or $\Delta P$, because it represents the polarization between the two memory states at a given time.

During irradiation, the capacitors top electrodes were shorted to the bottom electrodes. All samples were positively biased (by a read pulse) before each irradiation.

\section{RESULTS}

Previous results showed little change in the hysteresis loop polarization with extremely high levels of total dose [2]. The results of the present work are consistent with those earlier results. Figure 3 shows the hysteresis loops measured for dose levels up to $1.7 \mathrm{Mrad}\left(\mathrm{SiO}_{2}\right)$ (significantly less than the $100 \mathrm{Mrad}(\mathrm{Si})$ reported by Benedetto et al [2]). As can be seen from the figure, there is only slight degradation of the remanent polarization as a function of total dose, and there is no significant distortion of the loops. However, memory pulse test results for the same device show significantly more radiation damage. Figure 4 shows both the remanent polarization from the hysteresis loop test and

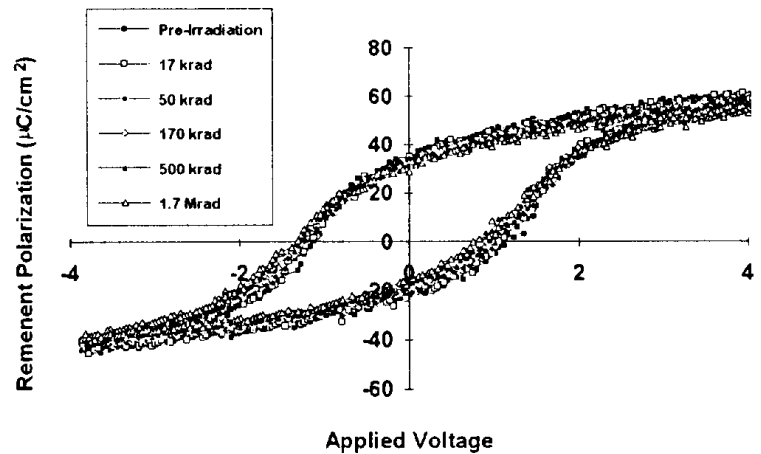

Figure 3: Hysteresis loops measured at each radiation dose show very little change due to irradiation.

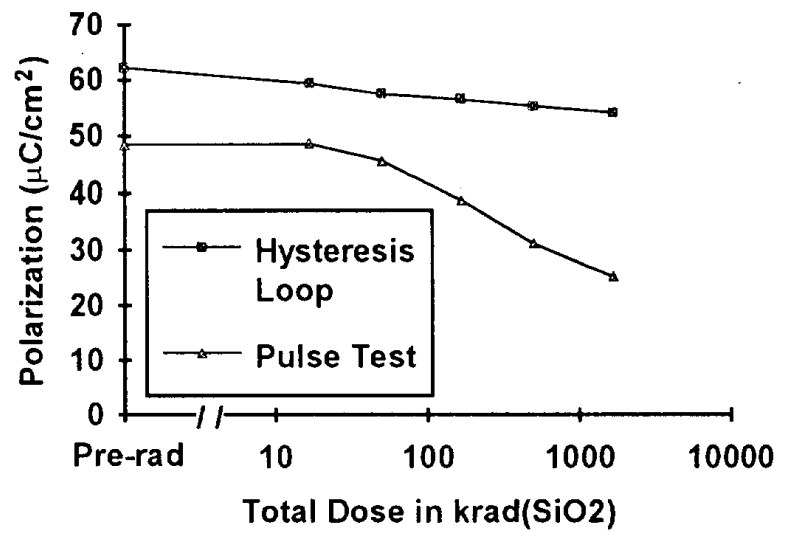

Figure 4: Remanent polarization from hysteresis loop measurements with pulse-retained polarization measurement results at each dose. The two measurements show significantly different levels of radiation-induced damage to the ferroelectric. 
the retained polarization from the pulse test for each total dose. The data in figure 4 show the remanent polarization from the hysteresis loop decreasing from about $62 \mu \mathrm{C} / \mathrm{cm}^{2}$ to about $54 \mu \mathrm{C} / \mathrm{cm}^{2}$ (about a 13 percent degradation), while the retained polarization measured by the pulse test fell from just under $50 \mu$ $\mathrm{C} / \mathrm{cm}^{2}$ to about $25 \mu \mathrm{C} / \mathrm{cm}^{2}$, a full 50 percent drop in retained polarization in "only" $1.7 \mathrm{Mrad}\left(\mathrm{SiO}_{2}\right)$. These data represent the average of the results from 6 capacitors.

To understand how these two measurements could yield such different results, it is important to recall that the hysteresis loop test measures a combination of the volatile and non-volatile components of the FE polarization, while the pulse measurement, at a 1-s retention time, only measures the non-volatile component. The differing responses by these two measurements then could occur two ways: (1) the ratio of volatile polarization to non-volatile polarization could have increased as a function of total dose, thereby allowing the hysteresis loop to continue to measure a relatively large signal, or (2) the hysteresis loop measurement itself could have caused a significant amount of annealing during the continuous sine wave measurement. (Note that the retained polarization measurement always preceded the hysteresis loop measurement).

The possibility of annealing due to cycling has been indicated in the results of earlier works $[2,3]$, but the hysteresis loop measurement in this work involved far fewer cycles than were used to intentionally anneal the samples in earlier works. During each hysteresis loop measurement the voltage on the sample was cycled for approximately $5 \mathrm{~s}$ at $10 \mathrm{kHz}$, for a total of 50,000 cycles at each dose. In earlier works approximately $10^{7} \mathrm{cycles}$ were applied before significant annealing was seen, but again hysteresis loops were being used to measure the polarization in these earlier works. It may be useful to think of two kinds of radiation damage (not necessarily two different mechanisms). The first kind is not removed by the hysteresis loop measurement (short term cycling) or by pulse testing (but can be removed by long term cycling, as seen in earlier works). The second kind is removed by hysteresis loop measurements but not by pulse testing. Since hysteresis loop measurements will not be used in a realistic non-volatile memory, the second kind of damage is not going to be removed in actual use. Therefore, it is important, when testing samples, not to neglect this damage by removing it with the test technique.

Since the total of the two components of polarization (volatile and non-volatile polarization as measured in the remanent polarization from the hysteresis loop) decreased only slightly (the $13 \%$ reported above), one would expect that the amount of volatile polarization has increased at the expense of non-volatile polarization.
That is, some of the non-volatile polarization may have become volatile polarization due to total dose ionizing radiation.

To examine the possibility that the ratio of volatile to non-volatile polarization has changed, we used a pulse retention test with time delays from $1 \mu \mathrm{s}$ to $100 \mathrm{~s}$ at each dose. This technique does not allow us to measure all of the volatile polarization directly; some of the switched polarization most likely decayed in less than $1 \mu \mathrm{s}$. (If one assumes that the hysteresis loop measurement correctly reports the sum of the volatile and non-volatile polarization, then the magnitude of the volatile component can be found by subtracting the nonvolatile component (from the pulse test) from the hysteresis loop polarization. We do not do this in this paper; here, we refer to the polarization that decays from $1 \mu \mathrm{s}$ to $1 \mathrm{~s}$ as the volatile component.) The results, shown in Figure 5, show a typical retention characteristic in which a significant amount of polarization (the volatile component) decays away (prerad). At about $1 \mathrm{~ms}$, the decay seems to be completed and the polarization stabilizes into a regime where it decays slowly and linearly in log time. Typically, the retention value at $1 \mathrm{~s}$ is used as a figure of merit for representing the long-term polarization storage of the material (non-volatile component). Before irradiation, this sample had an initial polarization at $1 \mu$ s of $49 \mu$ $\mathrm{C} / \mathrm{cm}^{2}$, which decays to $36 \mu \mathrm{C} / \mathrm{cm}^{2}$ at $1 \mathrm{~s}$. This is a loss of $13 \mu \mathrm{C} / \mathrm{cm}^{2}$ or 26 percent of the initial value. After irradiating to $1.7 \mathrm{Mrad}\left(\mathrm{SiO}_{2}\right)$, the sample had an initial polarization of $34 \mu \mathrm{C} / \mathrm{cm}^{2}$, which decays to $19 \mu \mathrm{C} / \mathrm{cm}^{2}$ at $1 \mathrm{~s}$. This is a loss of $15 \mu \mathrm{C} / \mathrm{cm}^{2}$ or 44 percent of the initial value. While the total magnitude of the decay increased very slightly, the change in the ratio of the volatile to non-volatile components was due primarily to the decrease in the non-volatile component.

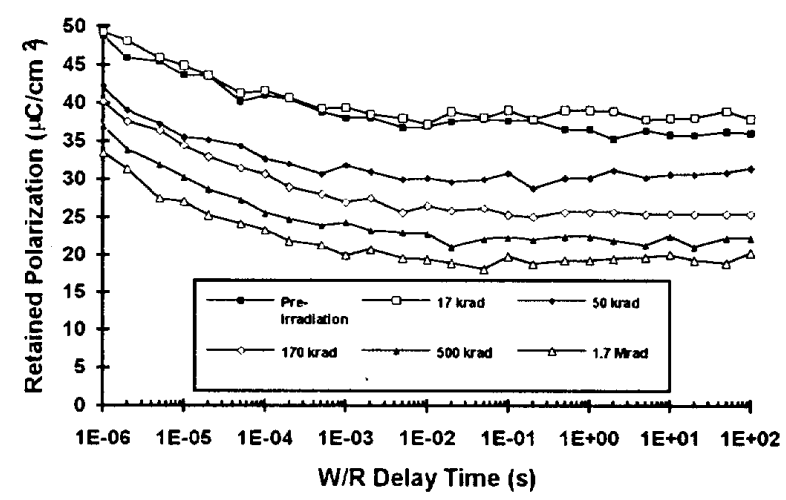

Figure 5: Retained polarization over a time range of $1 \mu \mathrm{s}$ to $100 \mathrm{~s}$ for each total dose showing the volatile polarization decaying from 1 $\mu \mathrm{s}$ to $1 \mathrm{~ms}$ and the non-volatile polarization remaining fairly constant afterward. This data is a representative set from one sample. 
To examine the second possibility, that the hysteresis loop measurement is annealing the damage done by the radiation, we performed another test sequence in which, at each dose level, a single 1-s pulse retention test was made, followed by a hysteresis loop measurement (the standard test sequence), and finally, a second 1-s pulse retention test. If the hysteresis loop measurement was removing the additional damage that showed up in the initial pulse test, the result of the second pulse test should follow the hysteresis loop curve exactly. If the hysteresis loop is removing none of the damage, the second pulse test should retrace the first pulse test. Figure 6 shows the results of this test, with each curve normalized to its initial value for a more direct comparison. The application of a hysteresis loop measurement before the pulse measurement significantly reduced the radiation damage; however, for the two highest dose levels, we still observed a significant amount of degradation. It remains to be seen whether additional cycling would anneal the remaining polarization decay. The data in Figure 6 represent the average results from 6 capacitors; the same capacitors were used in Figure 4.

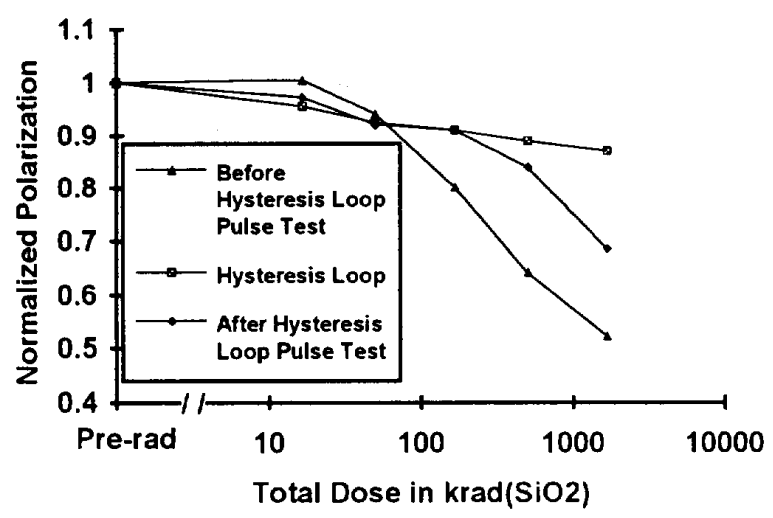

Figure 6: After each radiation dose, a $1 \mathrm{~s}$ retained pulse polarization measurement was made, then a hysteresis loop measurement, and finally another pulse polarization measurement. The results have been normalized to their pre-irradiation values.

The differing responses measured by the hysteresis loop and the pulse measurements appears to indicate a previously unknown mechanism, by which radiation damage impacts the retention of polarization (the nonvolatile component) in PZT ferroelectric thin films. Early predictions by Messenger and Coppage [9] indicated that charge deposited in the FE material by total dose radiation should not affect the FE polarization at doses of less then a few Mrad, and hysteresis loop data to date have supported this.

One possible explanation for this unexpectedly large degradation of the retained polarization may be the existence of internal "depoling fields" that are created when the FE polarization undergoes switching reversal. These fields cause the re-reversal of some of the switched polarization until the depoling fields are reduced below the coercive field (hence the reason for the pre-rad polarization decay). It may be that the addition of radiation-induced electron or holes trapped in the FE cause an increase in the magnitude of the depoling fields, and thus increase the amount of polarization that must reverse to compensate them. (The details of the internal depoling field model will be published in a separate journal article [10].)

We took an additional measurement of the pulse voltage characteristic to look for a shift in the coercive field due to total dose ionizing radiation. Figure 7 shows the result from a representative sample. In this measurement, the retained polarization is measured at $1 \mathrm{~s}$ for a range of voltages, from 1 to $8 \mathrm{~V}$, for each total dose. The results indicate that at all doses, the material begins significant switching between 1.5 and $2 \mathrm{~V}$. No change in the coercive field due to irradiation is indicated, but as expected, the retained polarization measured at each voltage decreases with dose. This implies that there is no net charge distribution across the film because (1) either the electrons or holes or both are equally distributed throughout the FE, or (2) the trapped electrons exactly balance out the trapped holes. Since neither Figure 3 or Figure 7 indicate and change in the materials coercive field, we assume no new permanent internal fields are being generated by this trapped charge. Therefore, the first case seems most reasonable.

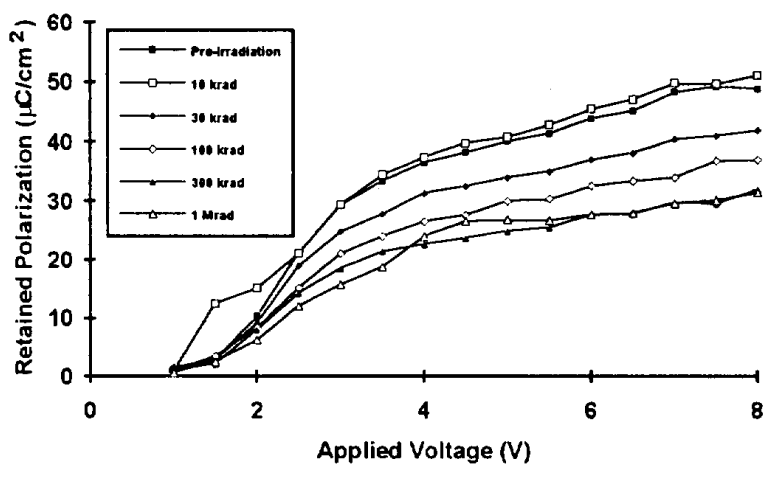

Figure 7: Voltage characteristic at each dose rate created by varying the amplitude of the applied write and read voltage from 1 to $8 \mathrm{~V}$. At all doses, the sample starts polarizing significantly at 1.5 to 2 $V$, which indicates no change in the FE coercive field. This data is a representative set from one sample. 


\section{CONCLUSIONS}

This paper examines the results of ionizing radiation on the retained polarization of PZT FE thin-films. We have presented data showing that ferroelectric materials are more sensitive to total-dose radiation damage than previously believed. We have shown that the nonvolatile component of the polarization decreased by 50 percent at only $1.7 \mathrm{Mrad}\left(\mathrm{SiO}_{2}\right)$. The non-volatile component is the polarization that is used for nonvolatile memory applications.

For these samples, hysteresis loop measurements underestimate the radiation sensitivity of FE films, because the hysteresis loop measurement may cause a significant part of the radiation damage to be annealed. By varying the write/read delay times, the pulse measurements allow a separation of the volatile and non-volatile polarization components for a better understanding of the radiation response of PZT. Although these results indicate that FE PZT may not be as radiation resistant as previously believed, the hardness of an unhardened FE NVRAM will undoubtedly still be determined by the hardness of the underlying circuitry.

\section{REFERENCES}

1. L. Kammerdiner and E. Philofsky, "Integration of Ferroelectric Capacitors into CMOS," presented at the International Symposium on Integrated Ferroelectrics (ISIF), Colorado Springs, CO (April 1993).

2. J. M. Benedetto, R. A. Moore, F. B. McLean, P. S. Brody, and S. K. Dey, "The Effect of Ionizing Radiation on Sol-Gel Ferroelectric PZT Capacitors," IEEE Trans. Nucl. Sci. NS-37, 1713-1717 (1990).

3. J. R. Schwank, R. D. Nasby, S. L. Miller, M. S. Rodgers, and P. V. Dressendorfer, "Total-Dose Radiation-Induced Degradation of Thin Film Ferroelectric Capacitors," IEEE Trans. Nucl. Sci. NS-37, 1703-1712 (1990).

4. S.C. Lee, G. Teowee, R.D. Schrimpf, D.P. Birnie, D.R. Unimann, and K.F. Galloway, "Total-Dose Radiation Effects on Sol-Gel Derived PZT Thin Films*," IEEE Trans. Nucl. Sci. NS-39, 2036-2043 (1992).

5. J. F. Scott, C. A. Araujo, H. B. Medows, L. D. McMillan, and A. Shawabkeh, "Radiation Effects of Ferroelectric Thin-Film Memories: Retention Failure Mechanisms," J. Appl. Phys. 66, 1444-1453 (1989).

6. T. F. Wrobel, J. A. Bullington and L. J. Schwee, "Radiation Hardness Evaluation of Thin Film Ferroelectric Capacitors," GOMAC Digest of Papers, 13, 267 (1987).

7. C. B. Sawyer and C. H. Tower, "Rochelle Salt as a Dielectric," Phy. Rev. 35, 269 (1930).

8. J. M. Benedetto, R. A. Moore, F. B. McLean, "FastDecay Component of the Remanent Polarization in Thin-Film PZT Capacitors," Integrated Ferroelectrics, Vol. 1, 195-204 (1992).

9. G. C. Messenger and F. N. Coppage, "FE Memories: A Possible Answer to the Hardened Nonvolatile Question," IEEE Trans. Nucl. Sci., NS-35, 1461-1466 (1988).

10. J. M. Benedetto, R. A. Moore and F. B. McLean, "The Effects of Operating Conditions on the Fast-Decay Component of the Retained Polarization in Lead Zirconate Titanate Thin-Films" accepted for publication in Journal of Appl. Phys., 1993. 